\title{
Perianal Reconstruction after Endometrioid Adenocarcinoma Excision using Inferior Gluteal Artery Perforator Flap
}

\author{
Burgic $\mathbf{M}^{1 *}$, Odobasic $\mathrm{A}^{2,3}$, Odobasic F ${ }^{3}$, Iljazovic $\mathrm{E}^{4}$, Odobasic $\mathrm{A}^{2}$ and \\ Mesic $\mathbf{M}^{2}$ \\ ${ }^{1}$ Clinic for Plastic and Maxillofacial surgery, University Clinical Center Tuzla, Bosnia \\ and Herzegovina
}

${ }^{2}$ Surgery Clinic, University Clinical Center Tuzla, Bosnia and Herzegovina

${ }^{3}$ Center of General and Colorectal Surgery-Odobasic, Tuzla, Bosnia and Herzegovina

${ }^{4}$ Clinic for Laboratory Diagnostics, Department of Pathology, University Clinical Center Tuzla, Bosnia and Herzegovina

*Corresponding author: Mufid Burgic, PhD, Clinic for Plastic and Maxillofacial surgery, Department of Plastic surgery, University Clinical Center Tuzla, Marsala Tita 60, 75000 Tuzla, Bosnia and Herzegovina, Tel: + 38761393153; Email: burgicmufid@yahoo.com

\section{Abstract}

Background: There are rare cases of endometrioid adenocarcinoma emerging from endometriosis of the uterine cervix in premenopausal woman.

Methods: We describe here a patient with this condition, presented with large perianal tumor, with a long-standing history of perianal endometriosis in episiotomy scar. This was initially evaluated with a Magnetic Resonance Imaging (MRI) and the diagnosis was confirmed by biopsy.

Result: The evolution of more wide excision surgery resulted in an increase in large defects of the perineal and perianal region. The inferior gluteal artery perforator (IGAP) flap was chosen as the best in a functional and aesthetic solution in this case.

Conclusion: The main recent advance in perianal reconstruction is the progress in perforator flap surgery. Reconstruction of perianal defects has functional and aesthetic considerations. Perianal reconstruction is very rare and review articles or larger case series are absent, hence only case reports have been published.

Keywords: Endometrioid adenocarcinoma; Episiotomy scar; Perianal reconstruction; IGAP flap

\section{Introduction}

The presence of endometrial tissue outside the uterus is defined as endometriosis [1]. The ovaries, uterine ligaments, rectovaginal and vesicovaginal septa, pelvic peritoneum, cervix, labia, vagina, and episiotomy scar are most commonly affected by endometriosis [2]. Although very rarely $(1 \%$ of cases $)$, complications, such as malignant transformation of endometriosis, may occur in women [3]. When treating conditions such as malignancy, perineal defects can frequently happen. Considering the complex anatomy of the perineum and the necessity to 


\section{International Journal of Transplantation \& Plastic Surgery}

preserve its function, these defects are very demanding to treat [4]. The same applies to perianal region, the reconstruction of which is presented in our case report.

Reconstructive techniques pose both functional and aesthetic challenges. To adequately reconstruct perineal defects, it is necessary to ensure the preservation of anogenital function and reconstruction, vulvovaginal reconstruction, no faecal or urinary contamination and skin coverage, while the dead space must be filled with vascularized tissue volume [5]. These also fall under fundamental conditions for the reconstruction of the perianal region.

The following report describes one case of perianal endometrioid adenocarcinoma in episiotomy scar treated by wide surgical excision. The aim is to present our longterm result of the use of inferior gluteal artery perforator (IGAP) flaps for the immediate reconstruction of the perianal region following excisional surgery.

\section{Case Report}

A 48-year old woman patient, without any family history of malignancy, was discovered an enlarged painful mass in the episiotomy scar with skin infiltration in the perianal area. The patient had an endometriosis surgery in 2000 and remained asymptomatic until November 2017 when she started complaining of general fatigue, anal discharge, anal bleeding, abdominal distension, and anemia. The patient underwent surgery in December 2017: hysterectomy and adnexectomy were performed via median laparotomy. During physical examination in January 2018, a firm nodule measuring approximately 30 x $20 \mathrm{~mm}$ was palpated in the episiotomy scar, intimately associated with vagina and anal canal (Figure 1).

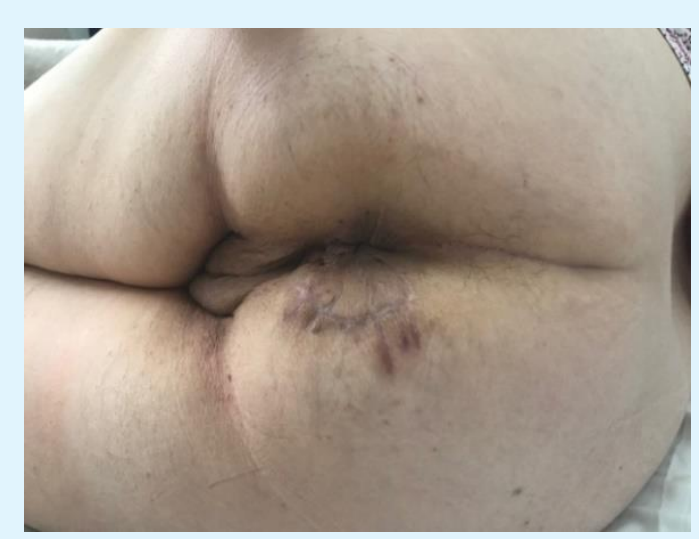

Figure 1: Preoperative status with firm nodule in the episiotomy scar.
After examination, the incision biopsy of tumor was performed. Histopathology revealed the foci of adenocarcinoma. Digital rectal examination did not reveal any mass in the anal canal or distal rectum, but the patient did have a sizeable tumor visible at the perianal skin.

The Magnetic Resonance Imaging (MRI) of the pelvis was performed to evaluate this mass better. The tumor measured $51 \times 32 \times 40 \mathrm{~mm}$ and appeared hyperintense on T2-weighted imaging. Anoscopy and rectoscopy examination results were normal. Internal pelvic organs showed as normal on gynecological and ultrasound examination. Endorectal ultrasonography showed a hyperechogenic mass extending along the right part of the anal canal. Rectal and anal wall, puborectal muscle, and anal muscle were unaffected, but the sphincter muscles were affected by the tumor. Small volume inguinal and pelvic lymph nodes were also visualized. Colonoscopy exam, which was performed next, confirmed rectal mucosa and anal canal were normal.

The surgery was performed under general anesthesia in the lithotomy or prone position. Wide excision of the tumor and the episiotomy scar was performed in February 2018. A large defect was effectively covered with Inferior Gluteal Artery Perforator flap (IGAP flap), using a very large flap supplied by two perforators. The tumor was precisely removed with partial resection of internal and external anal sphincter (Figure 2). First, reconstruction of anal sphincter was performed; perforators around the defect were identified and marked using a Doppler ultrasound. On the basis of the soft tissue defect and perforator localization, we decided upon the size of the flaps. These were made to be slightly larger than the defect. After skin incision, we elevated the flap at subfascial level. The IGAP flap was raised on two perforators, and intramuscular dissection was not necessary as we had sufficient flap mobility to cover the defect. In order to reduce the dead space and therefore the rate of postoperative complications, we deepithelialized medial part of the flap and introduced it into the place of previously removed tumor (Figure 3). All wounds were primarily closed tension-free. Two Redon high - vacuum drainages were inserted, and the same were removed on the third postoperative day. Result 1 month after treatment (Figure 4). The postoperative recovery period was satisfactory and without severe complications. The patient was discharged on the fifth postoperative day. One year after the operation, the patient was symptom-free with no signs of tumor recurrence. The histolopathological examination of the 


\section{International Journal of Transplantation \& Plastic Surgery}

tumor revealed an invasive endometrioid adenocarcinoma.

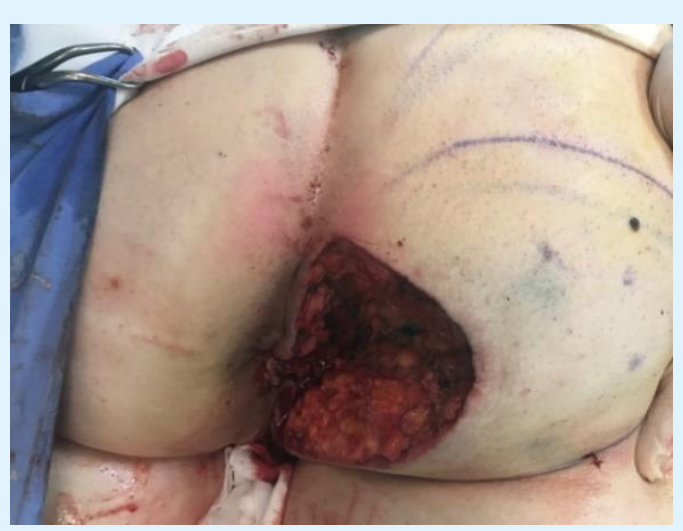

Figure 2: Radically excised tumor.

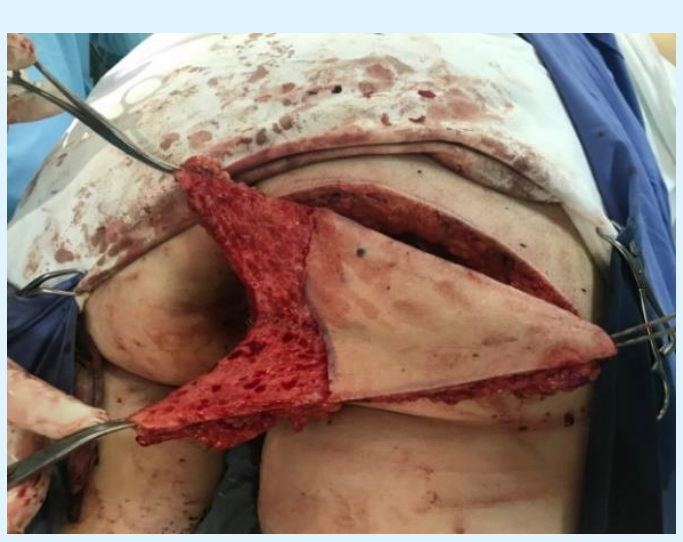

Figure 3: IGAP flap harvest for perianal reconstruction. The medial segment is deepithelialized and introduced into the defect.

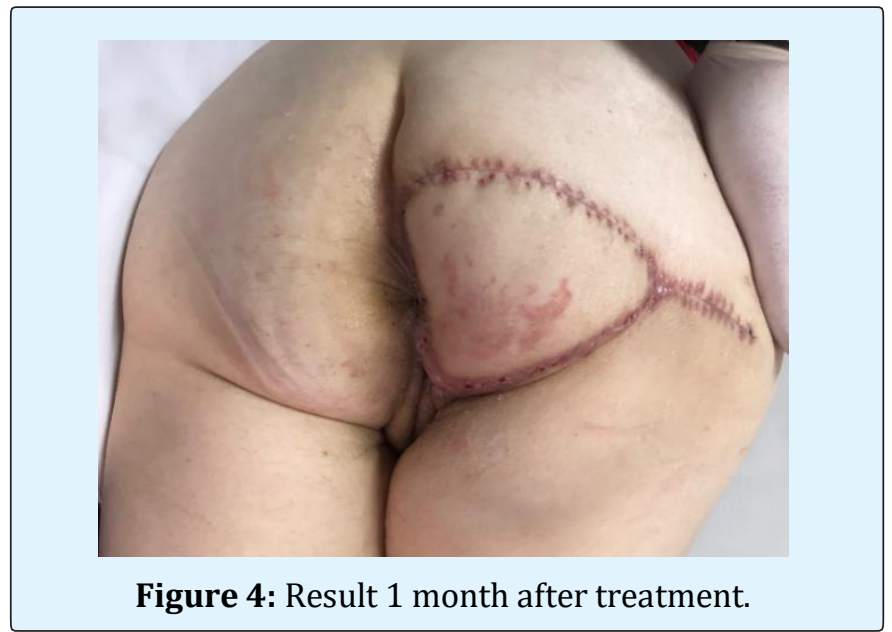

Present morphology with immunophenotype was consistent with moderate differentiated adenocarcinoma arising from endometriosis. Different immunophenotype verified in different morphologic pattern was consistent with mixed adenocarcinoma where endometrioid component consisted $40 \%$ and serous component with papillary growth consisted $60 \%$ of the tumor. Any transformation and progression from endometrioid to serous adenocarcinoma is also acceptable.

\section{Discussion}

Endometrial cancer ranks third among genital malignancy in women right after cervix and ovary cancers. Endometrioid adenocarcinoma, which affects 75$80 \%$ of patients, is the most common histological type of this cancer [6]. Malignant transformation of endometriosis associated with episiotomy scar is very rare [7]. Most surgical treatment options for successful reconstruction of perineum defects have been described in the literature. Reconstructive surgery for perineal, but also close perianal defects presents a difficult challenge because of the high rate of wound complications and tumor recurrence. The use of the perforator IGAP flap provides a better long-term result with successful repair.

Conventional flaps and free flaps may cause damage to the main veins, arteries and muscles, often causing secondary complications and additional procedures, as well as the morbidity of the donor site. On the other hand, perforator flap surgery has been considered a favorable method of reconstruction as it does not damage main blood vessels and muscles [8]. The study of Pramateftakis, et al. has shown that, after major excisions one should opt for IGAP flaps as a safe and reliable procedure to reconstruct the perineum [9]. Our case report has shown that the IGAP flap is suitable for perianal region because it was wider and thicker than the other flaps. Although radical treatments are the standards in these cases, they can often lead to large soft tissue defects.

The outcome of immediate reconstruction of the perianal region using IGAP flap is reduction of prolonged hospital stay. At any case, some diagnostic methods, including ultrasonography, magnetic resonance imaging, and biopsy, should precede the surgical approach. MRI is highly sensitive in detecting very small masses and offers excellent differentiation of endometrioid adenocarcinoma from neighboring tissue. A certain diagnosis can only be accomplished by histological examination of the tumor (biopsy), as was done in the case of our patient. 


\section{International Journal of Transplantation \& Plastic Surgery}

\section{Conclusion}

The main recent advance in perianal reconstruction is the progress in perforator flap surgery. Reconstruction of perianal defects has functional and aesthetic considerations. The inferior gluteal artery perforator (IGAP) flap was chosen as the best functional and aesthetic solution in this case, while overall patient's health and past treatments have also been considered.

\section{Compliance with Ethical Standards}

Conflicts of interest: The authors declare that they have no conflict of interest to disclose.

Human and Animal Right: This article does not contain any studies with human participants or animals performed by any of the authors.

Informed consent: For this type of study informed consent is not required.

\section{References}

1. Giudice LC, Kao LC (2004) Endometriosis. Lancet 364(9447): 1789-1799.

2. Testut L, Jacob O (1921) Traité d'anatomie topographique avec applications médicochirurgicales. Tome Premier: Téte-Rachis-CouThorax. Paris, France

3. Agarwal N, Subramanian A (2010) EndometriosisMorphology, Clinical Presentations and Molecular Pathology. J Lab Physicians 2(1): 1-9.
4. Wook Sung KI, Lee WJ, Yun IS, Lee WD (2016) Reconstruction of Large Defects in the Perineal Area Using Multiple Perforator Flaps. Arch Plast Surg 43(5): 446-450.

5. Mughal M, Baker RJ, Muneer A, Mosahebi A (2013) Reconstruction of perineal defects. Ann R Coll Surg Engl 95(8): 539- 544.

6. Kushner DM, Lurain JR, Fu TS, Fishman DA (1997) Endometrial adenocarcinoma metastatic to the scalp: case report and literature review. Gynecol Oncol 65(3): 530-533.

7. Ferrandina G, Palluzzi E, Fanfani F, Gentileschi S, Valentini AL, et al. (2016) Endometriosis-associated clear cell carcinoma arising in caesarean section scar: a case report and review if the literature. World J Surg Oncol 14(1): 300-304.

8. Kim JT, Ho SY, Hwang JH, Lee JH (2014) Perineal perforator-based island flaps: the next frontier in perineal reconstruction. Plast Reconstr Surg 133(5): 683-687.

9. Pramateftakis MG, Creasy H, Roblin P (2015) PTH287 7-year experience with perineal reconstruction following abdominoperineal or multivisceral resection using inferior gluteal artery perforator flaps. Gut 64(1): 537. 